PACS 61.05.cp, 61.10.Nz, 61.43.Bn, 61.72.Lk, 61,85. + P, 61.80.Cb

\title{
Calculated images of dislocations in crystals on section topograms
}

\author{
S.M. Novikov, I.M. Fodchuk, D.G. Fedortsov, A.Ya. Struk \\ Yuriy Fedkovych Chernivtsi National University,2 Kotsyubinskiy Str, 58012 Chernivtsi, Ukraine
}

\begin{abstract}
By means of numerical solution of the Takagi equations, modeling of X-ray topographic images of deformation fields of the dislocation loops and dislocation of different types. Diffraction images created by dislocations and dislocation loops of different size and spatial location are complicated and versatile in their thin structure.
\end{abstract}

Keywords: X-ray diffraction, topography, dislocation, dislocation loop, numerical simulation, image formation mechanisms.

Manuscript received 14.01.10; accepted for publication 08.07.10; published online 30.09.10.

\section{Introduction}

X-ray topography methods have gained wide acceptance for study of various defects in solids [1-3]. However, there is a problem related to interpretation of observed defect images (inverse problem solution). Various defects, being located in different positions with respect to scattering plane, can create diffraction images that practically do not differ.

With the advent of a variety of methods for digital processing of images in the experimental topograms [4], interest was rekindled to refinement of thin structure of topographic images of dislocations and their complexes $[5,6]$.

At the same time, using well-proven methods for numerical calculation of the Takagi equations [7-9] and achievements of elaticity theory in description of deformation fields created by defects, one can improve considerably the diagnostic possibilities of X-ray topography.

In this paper, by means of numerical solution of the Takagi equations, modeling of X-ray topographic images of deformation fields of the dislocation loops and edge dislocations is made depending on their spatial location in the scattering plane with respect to diffraction vector $\vec{h}$.

\section{Basic relationships}

If a crystal has inhomogeneities in the form of local or bulk-distributed elastic deformations, then X-ray wave field propagating in the crystal can be described by a system of the Takagi equations [1,7]. In the majority of cases this system of equations in partial derivatives is solved by numerical methods of finite differences $[1,8]$. For slowly varying amplitudes D0 and Dh in a crystal that has defects with a weakly varying distortion field $\left(\partial U_{k} / \partial x_{i}<<1\right)$, the combined Takagi equation is written as a relationship [7]:

$$
\left\{\begin{array}{l}
\frac{\partial D_{0}^{\prime}(\vec{r})}{\partial s_{0}}=-i \pi k C \chi_{h} D_{h}^{\prime}(\vec{r}) \\
\frac{\partial D_{h}^{\prime}(\vec{r})}{\partial s_{h}}=-i \pi k\left[C \chi_{h} D_{0}^{\prime}(\vec{r})-2 \alpha(\vec{r}) D_{h}^{\prime}(\vec{r})\right]
\end{array},\right.
$$

where $\chi_{0}, \chi_{h}$ are the Fourier components of polarizability.

The local misorientation function $\alpha(\vec{r})$ is proportional to projection of gradient from scalar product of diffraction vector $\vec{h}$ and displacement vector $\vec{U}$ to direction of diffracted rays propagation $\vec{s}_{h}$ :

$\alpha(\vec{r}) \sim \vec{s}_{h} \cdot \vec{\nabla}(\vec{h} \cdot \vec{U})$,

For an isotropic medium in the case of placement of the dislocation line along the $\mathrm{Oz}$ axis expression for the dislocation is given in [16]:

$$
\begin{aligned}
\vec{U}(\vec{\rho})= & \frac{\Omega}{4 \pi}\left(-\vec{b}^{\prime}-\frac{1-2 v}{1-v}\left[\vec{t}^{\prime} \times \vec{b}^{\prime}\right] \ln \rho+\right. \\
& \left.+\frac{1}{1-v} \frac{\left[\vec{t}^{\prime} \times \vec{b}^{\prime}\right] \cdot \vec{\rho}}{\rho^{2}} \vec{\rho}\right),
\end{aligned}
$$

where $\Omega$ - the solid angle of the observation point, which limits the dislocation segment is selected for modeling, $\vec{\rho}$ - radius vector, $v$ - Poisson's ratio.

Note that the combined equation (1) in the case of $\left(|\alpha(\vec{r})|>\left|\chi_{0}\right|\right)$ is easily transformed into a kinematic one. Areas with different signs of $\alpha(\vec{r})$ functions differently 
deflect strongly and weakly absorbing Bloch waves [1]. From the appearance of intensity rosette $R(x, y)$ one can reproduce local misorientation rosette of $\alpha(\vec{r})$ defect and determine the sign of the Burgers vector [1,10-12].

\section{Simulation results}

\subsection{Dislocation loops}

Typical for silicon are the simplest dislocations whose Burgers vector equals $\vec{b}=a / 2<110>$ [15], i.e.: screw dislocation; $60^{\circ}$ dislocation; edge dislocation located in slip plane $\{100\}$; and dislocation loops. Representation of a dislocation loop as a hexagon, whose segments are oriented along the $<110>$ directions $\left(60^{\circ}\right.$ dislocations $)$, and Burgers vectors $\vec{b}=a / 2<110>$ of each dislocation segment lie in the loop plane, is apparently the most probable model presentation of sliding dislocation loop $[13,14]$.

However, the simplest model of a dislocation loop (DL) can be constructed in the form of a spherically symmetric inclusions of different sizes $\left(R_{p}\right)$ and powes $\left(C_{v}\right)$. Geometrically such loop can be represented as a circle (Fig. 1) [13,17].

Modeling of X-ray diffraction images of sliding DL (Fig.2) was made for $\mu t \approx 1$ and $\mu t>10$ ( $\mu$ is photoelectric absorption coefficient), crystallographic orientation of entrance surface (111), (440) reflection of $M o K_{\alpha^{-}}$and $\mathrm{Cu} K_{\alpha}$-radiation in the diffraction geometry according to Laue. Under diffraction conditions $\mu t>10$ the case of anomalous transmission of X-rays is realized (Fig.2b).

Fig. 3 shows the calculation of images was performed for prismatic loop, located at an angle to the crystal surface (110), where $\vec{b}=a / 3[1 \overline{1} \overline{1}], \vec{b}=a / 3[\overline{1} 11]$, which is typical of Frank dislocation loop [14].

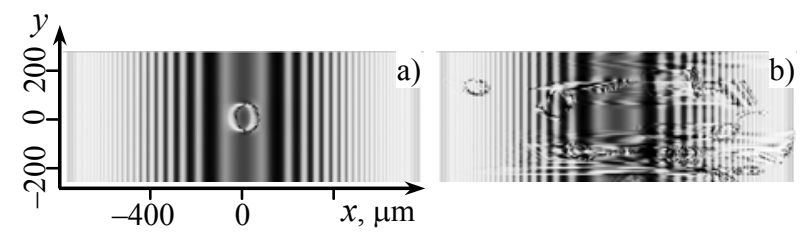

Fig. 1.. Section topograms of dislocation loops. Si, (440) $\mathrm{Mo} K_{\alpha}, \mu t=3 . C_{v}=10^{-16}, R_{p}=50 \mu \mathrm{m}$. a) Depth of occurrence is $1800 \mu \mathrm{m}$, б) arbitrary (random) distribution in the volume.
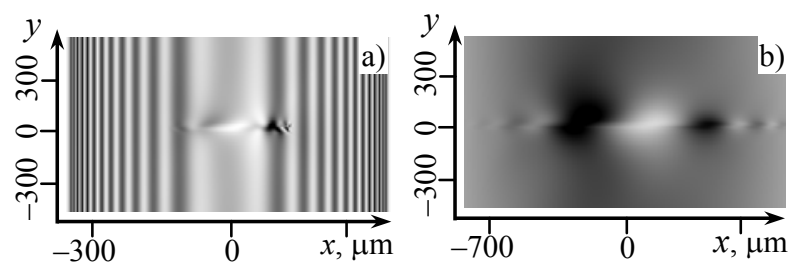

Fig. 2. $R(x, y)$ of dislocation loop at the distance of $300 \mu \mathrm{m}$ to exit surface obtained by means of (440) $\mathrm{MoK}_{\alpha^{-}}, \mu t=1,2$ (a) and $\mathrm{Cu} K_{\alpha}$-radiation $\mu t=12$ (b).

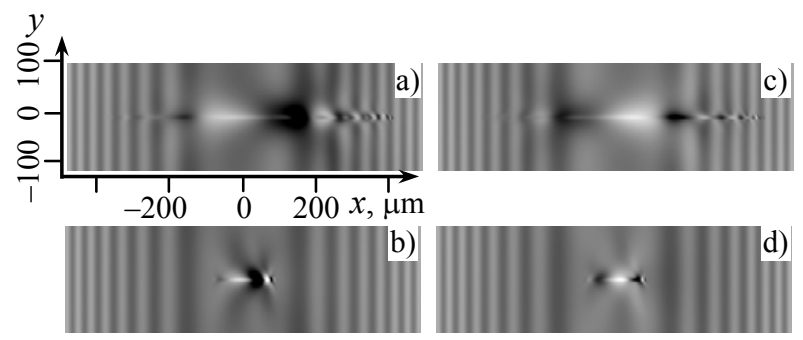

Fig. 3. $R(x, y)$ of prismatic dislocation loops $(D=10 \mu \mathrm{m})$ : $z=1000 \mu \mathrm{m}(\mathrm{a}, \mathrm{c}), z=1800 \mu \mathrm{m}(\mathrm{b}, \mathrm{d}), \mu t=3 . \vec{b}=1 / 3[1 \overline{1} \overline{1}](\mathrm{a}, \mathrm{b})$ and $\vec{b}=1 / 3[\overline{1} 11](\mathrm{c}, \mathrm{d})$. Occurrence plane $(\overline{1} 11)$.

If the crystal thickness, where the DL is placed, is large enough then only one weakly absorbing Bloch wave comes to it. In strongly distorted defect region $\left(\alpha(\vec{r})>>\left|\chi_{h}\right|\right)$ a weakly absorbing Bloch wave is subject to interband scattering [1]. As a result, a new wave filed is excited that is composed of two types of Bloch waves. At point of existence of both Bloch waves their interference takes place and as a result extinction intensity modulations can be observed (Fig.3).

Depending on the plane of dislocation loop occurrence and its type, diffraction image can be changed not only the distribution of black-white contrast

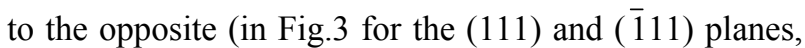
respectively), but it also become considerably weaker or stronger. On the whole, except for separate details, this variety of DL diffraction images depends on the contribution of scalar products $(\vec{h} \vec{b})$ of individual dislocation loop segments to total local misorientation function $[1,17,19]$, whereas DL occurrence plane and its orientation with respect to diffraction plane are "hidden" in these products.

\subsection{Inclined to the surface $72^{\circ}$ and $60^{\circ}$ dislocations}

In the case of inclined to the entrance crystal surface dislocations (Fig.4, 5) intensity modulations, which caused by overlapping of normal and abnormal refracted Bloch waves are observed on topograms. For all possible cases of placing the input and output points of dislocations through the Bormann fan, the section images of dislocation, which parameters are defined by the spatial orientation of the Burgers vector with respect to the diffraction vector, are obtained. Crucial for the formation of a clear contrast for $72^{\circ}$ dislocation in Fig.4 is a cross section of the Bormann fan in the direction of incidence X-ray $\vec{s}_{0}$.

As the distance from the dislocation location to the exit surface is increased, X-ray diffraction image of dislocation expands due to divergence of rays within Bormann fan. As a result the interferention interaction of newly created and existing wave fields is weakened. 
In the case of $\mu t=1$, the positive contrast region (dark relatively background) around dislocations begins where there is a noticeable interbranch scattering of Bloch X-wave. Depending on the geometry of location of dislocation and its orientation characteristics interference fringes can take diferent forms. For the case of dislocation lines inclined orientation relative to the diffraction vector in the plane of scattering (Fig. 4), interference pattern on appearance reminds sectional image of the inclined stacking fault [1]. A somewhat different situation is observed for $60^{\circ}$ dislocation in fig.5. Its image essentially depends on where the dislocation is located - near the entrance, in the center or far from the exit surface of the crystal.

Overall, the picture of display of interbranch scattering essentially depends on the type of defect. In particular, for thick crystal on fig.4c under the formation of dislocation image dynamic part of the image dominates, while its direct (kinematic) image is hardly noticeable.

Direct image of the sectional topographs (Fig.4, 5) is formed by the crystal with a few microns thickness, while the misorientation value of reflecting planes in this area is about twice bigger than the width of the reflection curve of a perfect crystal. This image is formed mainly by the kinematic scattering with indirect influence of dynamic effects.

The basic law of contrast change and the formation of diffraction images in the case of projection and section topography for thin $(\mu \mathrm{t}=1)$ and $\operatorname{thick}(\mu \mathrm{t}=10)$ crystals are the same [1]. However, the thin structure of image is very different: the intensity oscillations are less contrast.
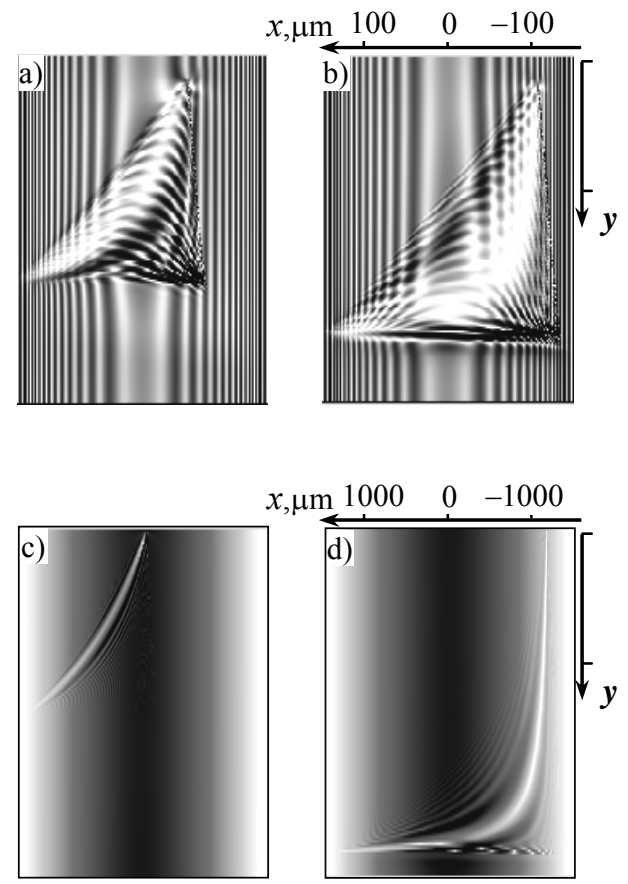

Fig. 4. Section topograms of inclined $72^{\circ}$ dislocations: $\mathrm{Si}$, $\operatorname{MoK}_{\alpha} \quad(220), \mu t=1 \quad(a, b), \mu t=10 \quad(\mathrm{c}, \mathrm{d})$. Output point of dislocations to the exit surface is at the center $(\mathrm{a}, \mathrm{c})$ and closely to the edge of the Borrmann fan $(b, d)$.
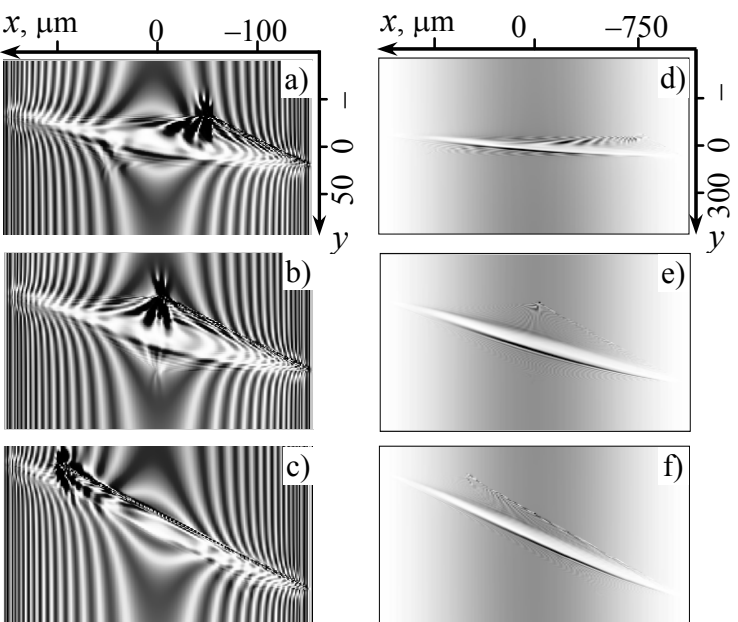

Fig.5. Section topograms of $60^{\circ}$ dislocations, whih are parallel to the exit surface. Si, $\mathrm{Mo}_{\alpha},(220): \mu t=1$ (a-c), $\mu t=6$ (d-f). The depth of location : a) $z=240$, b) 360 , c) 600 , d) 500, e) 2000 , f) $3000 \mu \mathrm{m}$.

\subsection{Edge dislocation with a dislocation line perpendicular to the sample surface and Burgers vector parallel to diffraction vector}

The features of formation of images in section topographs of edge dislocations that are perpendicular to the surface of the crystal are investigated. This orientation of dislocations is interesting that the function $\alpha(\vec{r})$ does not depend on the thickness of the crystal and the formation of $\mathrm{X}$-wave diffraction image is due to specific effects of channeling [3,18].

Analysis of the calculated and experimental topograms (Fig. 6) reveals some features of formation of the thickness oscillations (Fig. 7) and topographic images of elastic deformation fields in a strongly distorted crystal regions. In general, the distribution of intensities of $R_{h}(x, y)$ in the large deformation areas repeats a function of $\partial \alpha(x, y) \partial x$ (Fig. 6a,c). From the analysis of topograms on Fig. 6, it follows that scattering areas with large intensity are observed in places where changing of values of the strain is non-significant along the diffraction vector (the central region of topogram).

However, in the crystal region where deformations are changing rapidly, "specific" effects of channeling Xwaves along the reflecting planes are completely inhibited.

On the distributions $R_{h}(x, z)$ (Fig. 7a-c) as distance from the gliding plane the asymmetrical inhibition of extinction oscillations of intensity in the base of Bormann fan and intensity concentration in the center are occur. In fact a quasi-periodic standing wave with macroperiod order of $7-10 \Lambda^{\sigma}$ which extends as through the waveguide in large values of function $\alpha(x, y)$ is arising.

The amplitude of new intensity oscillations at the thickness distributions in the scattering plane in the areas of tensile strain under dislocation extra plane dramatically increases when approaching to the line of dislocation (Fig.7a). 


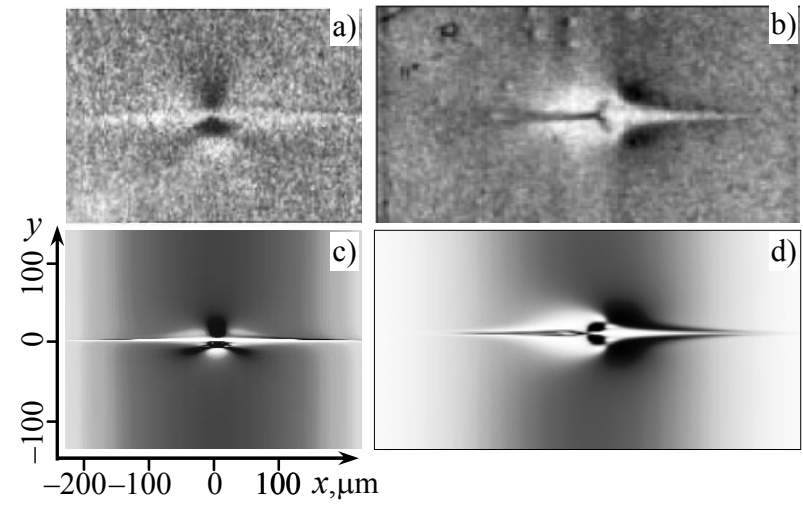

Fig. 6. Section topograms of edge dislocations: $\mathrm{Si}, \mathrm{Mo} K_{\alpha 1^{-}}$ radiation: a), c) (220); b), d) (004). (a, b) - experimental image [5], (c, d) - calculated image.
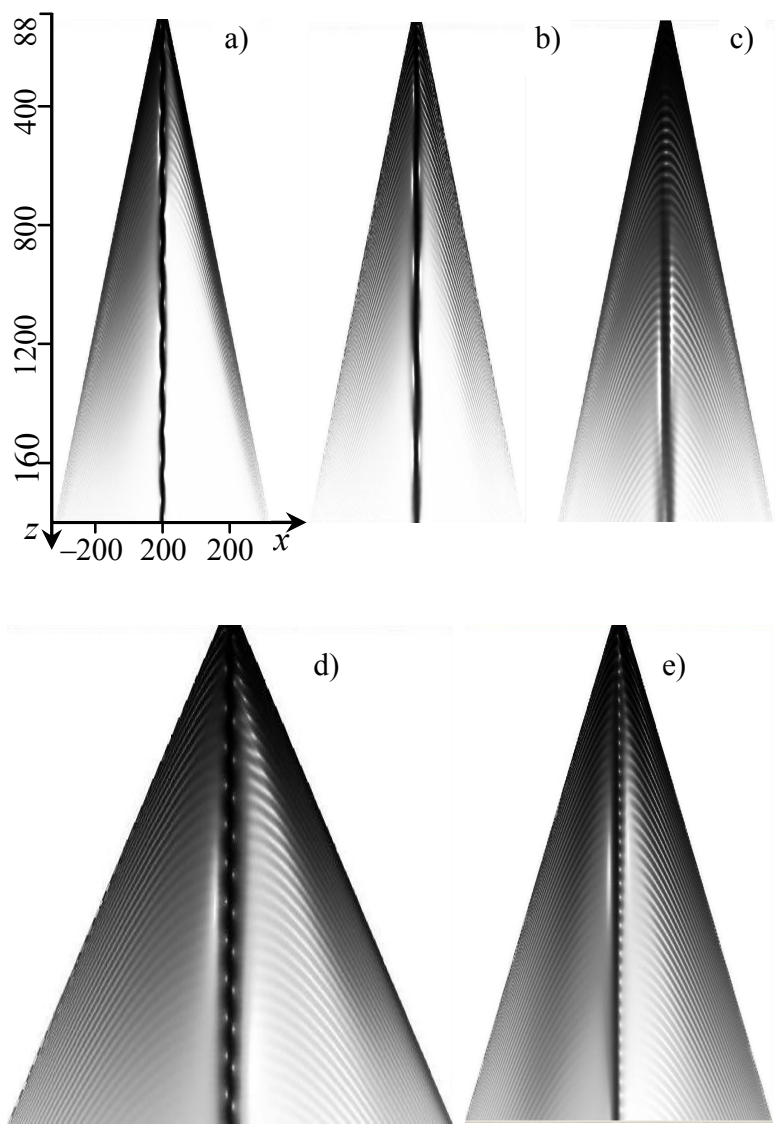

Fig. 7. Distributions $R_{h}(x, z)$, where indent from the line of dislocation parallelly to the glide plane is equal to $y_{0}=1(\mathrm{a}, \mathrm{d}$, e), $y_{0}=-5$ (b), $y_{0}=-10 \mu \mathrm{m}$ (c) for $\mathrm{MoK}_{\alpha 1}$-radiation. ( $\left.2 \overline{2} 0\right)$ (a-c), (4 40$)(d),(004)(e)$.

Period of new intensity oscillations exceeds the extinction period $\Lambda$ (5-10 times) and non-linearly increases with decreasing of value

$$
\alpha(x=0, y)=-\frac{\sin (\theta)(\vec{h} \vec{b})}{|\vec{k}| \pi}\left(\frac{(1+2 v)}{2(1-v)}\right) \cdot \frac{1}{y} .
$$

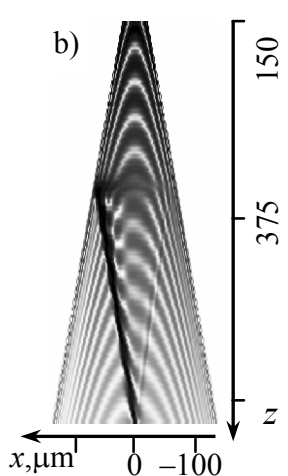

Fig. 8. Section topograms of diffraction contrast from screw dislocation $\mu t \sim 1, \operatorname{Mo} K_{\alpha}$ (220) respective diffraction sections (a) and planes $(y=10 \mu \mathrm{m})(\mathrm{b})$.

This is due to increase of the effective thickness of the crystal, where accumulation of the phase of newly interfering fields in the strongly deformed crystal region is equal to $2 \pi$. The same pattern is observed in the area of compressive strain (above the glide plane). At the same time period and amplitude of mode oscillations are significantly lower. On the topographs in tension areas under the extra plane the dark (positive to the background) contrast is formed.

Similar regularities of intensity propagation along the dislocation line are inherent and for other reflections (fig. $7 \mathrm{~d}, \mathrm{e})$.

\subsection{Screw dislocation}

In Fig. 8 diffraction image for screw dislocations, whose axis is oriented parallel to the diffraction vector is given. Depth of occurrence of dislocations is equal to half the thickness of the crystal $\mu t=1$. This case of the orientation of dislocation is also "special", since the displacement of the reflecting planes $\vec{u}_{d}$ do not depend on coordinate $x$.

Features and mechanisms of formation of diffraction images for described case of screw dislocation orientation are well studied in $[1,8]$.

In [19] the features of the impact of acoustic strains on formation as X-ray topographic screw dislocation contrast in general, and the formation extinction oscillations in particular are investigated. It is noted that with a small acoustic deformations significant changes in image of screw dislocations occur near the output point of kinematic image on the surface of the crystal.

\section{Conclusions}

1. The variety of X-ray images of dislocation loops on section topographs depending on crystalographic and spatial location was explaned by the combined contribution of products $(\vec{h} \vec{b})$ of each of the loop segments to total local misorientation function $\alpha(\vec{r})$. 
2. The features of formation of the diffraction images on section and projection topograms of screw, edge, $60^{\circ}$ and $72^{\circ}$ dislocations depending on their location in Bormann fan in thin and thick crystal were established.

3. In the case of a special (perpendicular to surface) location of edge dislocations in the Bormann fan specific effects of channeling and complete external reflection of $\mathrm{X}$-waves were observed. In strongly distorted crystal region near the dislocation line the spatial quasi-periodic wave field with macroperiod in several extinction distances $\Lambda^{\sigma}$ which extends as through the waveguide in large values of function local misorientations $\alpha(x, y)$ is arising.

\section{References}

1. A. Authier, Dynamical Theory of X-Ray Diffraction. Oxford University Press, New York, 2001.

2. D.K. Bowen, B.K. Tanner, High Resolution X-Ray Diffractometry and Topography. Taylor Francis, London, 1998.

3. E.V. Suvorov, I.L. Shulpina, X-Ray Optics of Defect Crystals // Surface No.7, pp. 3-32 (2001) (in Russian).

4. L.N. Danil'chuk, V.A. Tkal', A.O. Okunev, Yu.A. Drozdov, Digital processing of X-ray topographic and polarization-optical images of single crystal structure defects. Y. Mudryi Novgorod State University, Velikyi Novgorod, 2004 (in Russian).

5. E.V. Suvorov, I.A. Smirnova, E.V. Shulakov, Formation of the image of an edge dislocation in an absorbing crystal // FTT 49(6), pp. 1050-1057 (2007) (in Russian).

6. J. Hartwig, Hierarchy of dynamical theories of $\mathrm{x}$ ray diffraction for deformed and perfect crystals // J. Applied Physics. 34(10A), pp. A70-A77 (2001).

7. S. Takagi, Dynamical theory of difraction applicable to crystals with any Kind of small distortion // Acta Cryst. 15, pp.1311-1312 (1962).
8. Y. Epelboin, Simulation of X-ray topografs // Material Science and Engineering 73, pp.1-43 (1985).

9. Y.Epelboin, A varying-step algorithm for the numerical integration of Takagi-Taupin equations // Acta Cryst. A37, pp.132-133 (1981).

10. V.L. Indenbom, V.M. Kaganer, The formation of plane-wave X-ray images of microdefects // Phys. status solidi A 87, pp. 253-265 (1985).

11. L.N.Danil'chuk, Рентгеновское наблюдение полей деформаций вокруг краевых дислокаций в монокристаллах кремния // FTT 11, pp. 3085 3091 (1969) (in Russian).

12. L.N.Danil'chuk, Ju.A.Drosdov, A.O.Okunev et. al., $\mathrm{X}$-ray topography of single crystal structure defects in semiconductors on the basis of the Borrmann effect (review) // Factory Lab. Diagnostics of Materials 68(11), pp. 24-33 (2002) (in Russian).

13. C. Teodosiu, Elastic Models of Crystal Defects. Mir, Moscow, 1985 (in Russian).

14. J.P.Hirth, J. Lothe, Theory of Dislocations. Eds.: Atomizdat, Moscow, 1972 (in Russian).

15. R.G.Rodes, Imperfections and active centres in semiconductors. Metallurgiya, Moscow, 1968 (in Russian).

16. R. De Wit, Some Relations for Straight Dislocations // Phys. Stat. Sol. 20, pp.567-573 (1967).

17. V.B. Gevyk, S.N. Novikov, D.G. Fedortsov, I.M. Fodchuk, Simulation of X-ray diffraction images of dislocation loops in crystals // Met. Phys. Adv. Tech. 27(9), pp.1237-1250 (2005) (in Russian).

18. A. Authier, F. Balibar, Création de Nouveaux Champs d'Ondes Dus à la Présence d'un Objet Diffractant à I'Intérieur d'un Cristal Parfait. II. Cas d'un Defaut Isole // Acta Cryst. A26, pp.647-654 (1970).

19. S. Novikov, I. Fodchuk, D. Fedortsov, A. Struk, Xray section images of dislocations and dislocation barriers in Si // Phys.stat.sol.(a) 206(8), pp. 18201824 (2009). 